\title{
Welding molecules into polymeric chains in one fell swoop
}

\author{
Longyu Li and Chenfeng $\mathrm{Ke}^{*}$
}

Molecular components that are not covalently linked but topologically interlocked represent a family of molecules named mechanically interlocked molecules (MIMs) [1]. One archetype of MIMs is catenane (the Latin "catena" means "chain"), which is formed between two ([2]catenane, Fig. 1a) or more ([n]catenane) mechanically interlocked rings. Similar to their macroscopic counterpart, i. e., interlocked metal chains, these molecular ring components can move and deform (Fig. 1b) in response to external stimuli. Theoreticians have projected [2] poly $[n]$ catenanes with high flexibility and toughness, which are often contradicting properties to be obtained in polymeric materials. The syntheses of catenates and polycatenanes represent grand challenges. The first [2] catenane was obtained serendipitously with less than $1 \%$ yield in the 1960s [3]. Sauvage (2016 Nobel laureate) opened the door for practically synthesizing oligo-catenanes via a metal templated ring closing strategy (Fig. 1a) [4]. Since then, more complex catenane architectures, e.g. [5]catenane [5] and [7]catenane [6], have been successfully prepared through a stepwise ring closing method. Taking catenanes a step beyond molecules and to the polymeric world is even more challenging-stepwise welding or acyclic molecules into rings is impractical for polymer chemists, imaging closing an Avogadro number of ring components one-by-one! Writing in Science, Rowan and coworkers from the University of Chicago reported [7] a metal templated synthesis of poly $[n]$ catenanes containing linear poly[7-26]catenanes, cyclic poly[4-7]catenanes and branched poly[13-130]catenanes to realize this long-standing dream.

Encouraged by their previous accomplishment [8] on the efficient ring-closing synthesis of a [3]catenane, in this work, the authors designed a 68-membered ring component (R1) and a 63-membered C-shaped acyclic ring precursor (AR2) for the poly $[n]$ catenane synthesis.
In the presence of a $\mathrm{Zn}^{2+}$ template, a supramolecular copolymer linked by $\mathrm{Zn}^{2+}$ and the Bip ligands (Fig. 1c) of $\mathbf{R 1}$ and AR2 was formed in solution. After ring-closing metathesis, metal coordinated poly $[n]$ catenane mixtures were obtained, which was demetalated subsequently. Although the synthesis of their previous [3]catenane is highly efficient, taking this approach and applying it to the poly $[n]$ catenane face many technical obstacles-the purification and characterization to confirm the mechanically interlocked architecture are not trivial, because any ill-defined AR2-to-ring transformation will lead to undesired side products. The authors cleverly took advantage of the catenane effect [9] to selectively re-metallate the obtained interlocked polymers with $\mathrm{Zn}^{2+}$, removing the non-interlocked byproducts by solid-liquid extraction. The isolated poly $[n]$ catenanes were further separated into branched poly[13-130]catenates, linear poly[7-27]catenates, and cyclic poly[4-7]catenanes at a ratio of 28:61:11 (Fig. 1c) using gel permeation chromatography. The average numbers of the chain end $\left(N_{c}\right)$ of the correspondent poly $[n]$ catenanes were calculated based on the measured absolute molecular weight and NMR chain-end analysis to identify the interlocked topology (Fig. 1c) - cyclic poly $[n]$ catenanes with no chain end $\left(N_{c}=0\right)$, linear poly $[n]$ catenanes containing two ends $\left(N_{c}=2\right)$, and branched ones possessing more than 4 chain ends. Interestingly, linear poly $[n]$ catenanes exhibit $70 \%$ hydrodynamic size expansion after $\mathrm{Zn}^{2+}$ metalation with significantly increased glass transition temperature, attributed to the constrained ring conformations in those linear poly $[n]$ catenanes. The hydrodynamic size expansion of the cyclic and branched poly $[n]$ catenanes were smaller compared to those of the linear ones after metalation due to their topological difference (Fig. 1d).

In this work, Rowan et al. paved the way for the first practical synthesis, isolation and characterization of poly 
a Metal-templated catenane synthesis

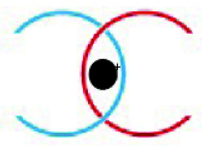

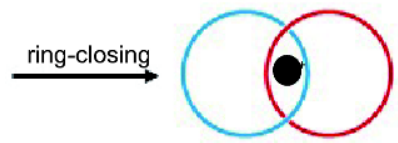

b Comformational motions of catenanes
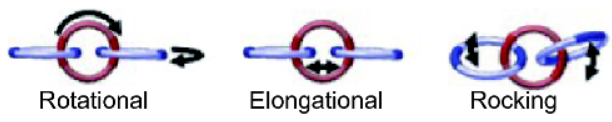

C Poly[n]catenane synthesis reported in this work
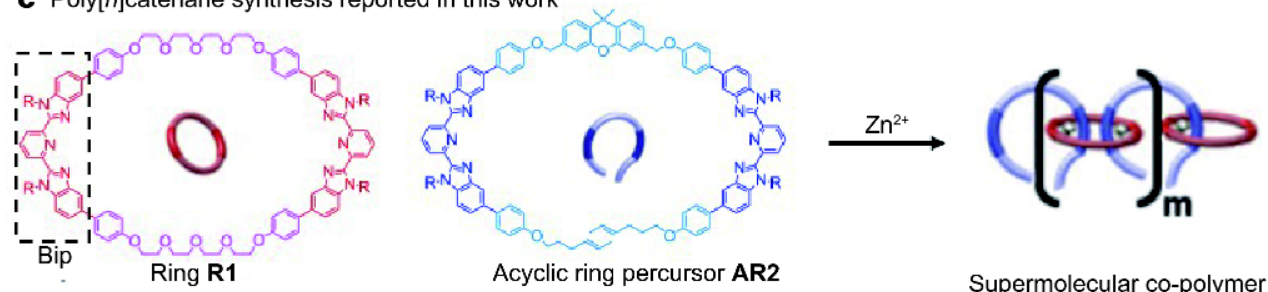

Acyclic ring percursor AR2

Supermolecular co-polymer

d

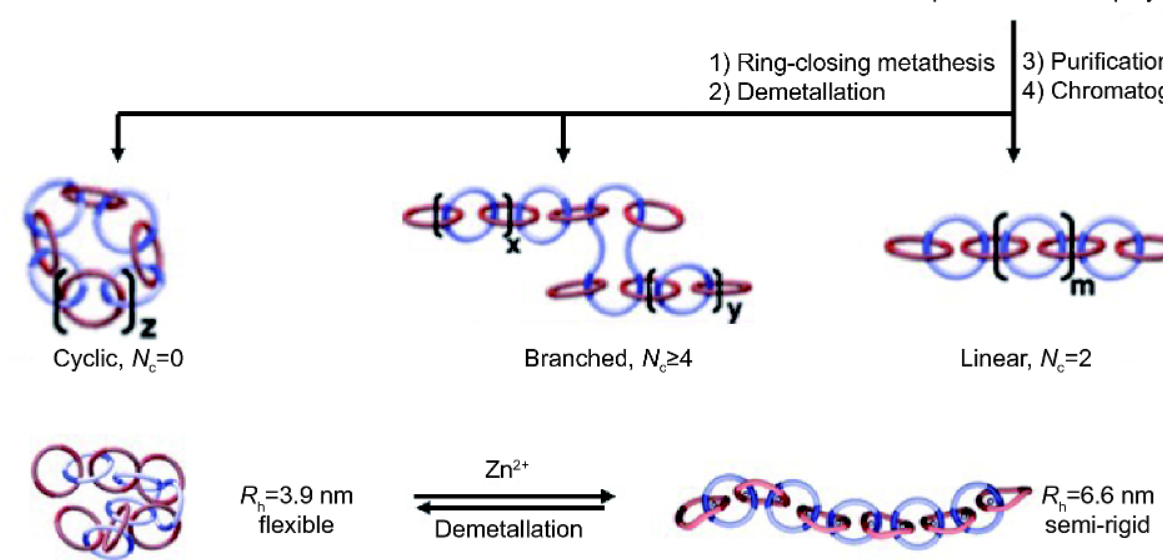

Figure 1 (a) Metal-templated synthesis [4] of a [2]catenane by forming a metal complex followed by ring closing. (b) Possible ring motions in a [3] catenane. (c) Metal-templated synthesis of poly $[n]$ catenane [7] using ring $\mathbf{R} \mathbf{1}$ and acrylic ring precursor $\mathbf{A R 2}$. In the presence of $\mathrm{Zn}{ }^{2+}$, these precursors form metal-templated supramolecular co-polymer in solution, and the C-shaped AR2 was subsequently closed to form linear, branched and cyclic poly $[n]$ catenanes. (d) Hydrodynamic radius $\left(R_{\mathrm{h}}\right)$ change of a linear poly $[n]$ catenane upon (de)metalation. Reprinted with permission from Ref. [7]. Copyright 2017, American Association for the Advancement of Science.

$[n]$ catenanes. Upon (de)metalation, these poly $[n]$ catenanes exhibit very different hydrodynamic size changes related to their topological architectures. With real poly $[n]$ catenane materials in hand, it is intriguing to chemists to understand their performance in bulk related to the interlocked topologies for the future development of smart materials.

Received 28 December 2017; accepted 29 December 2017; published online 12 January 2018

1 Bruns CJ, Stoddart JF. The Nature of the Mechanical Bond: From Molecules to Machines. John Wiley \& Sons. 2016

2 Pakula T, Jeszka K. Simulation of single complex macromolecules. 1. structure and dynamics of catenanes. Macromolecules, 1999, 32: $6821-6830$
3 Wasserman E. The preparation of interlocking rings: a catenane. J Am Chem Soc, 1960, 82: 4433-4434

4 Dietrich-Buchecker CO, Sauvage JP, Kintzinger JP. Une nouvelle famille de molecules: les metallo-catenanes. Tetrahedron Lett, 1983, 24: 5095-5098

5 Amabilino DB, Ashton PR, Reder AS, et al. Olympiadane. Angew Chem Int Ed, 1994, 33: 1286-1290

6 Amabilino DB, Ashton PR, Boyd SE, et al. The five-stage selfassembly of a branched heptacatenane. Angew Chem Int Ed, 1997, 36: 2070-2072

7 Wu Q, Rauscher PM, Lang X, et al. Poly $[n]$ catenanes: Synthesis of molecular interlocked chains. Science, 2017, 358: 1434-1439

8 Wojtecki RJ, Wu Q, Johnson JC, et al. Optimizing the formation of 2,6-bis(N-alkyl-benzimidazolyl)pyridine-containing [3]catenates through component design. Chem Sci, 2013, 4: 4440-4448

9 Lewis JEM, Galli M, Goldup SM. Properties and emerging applications of mechanically interlocked ligands. Chem Commun, 2016, 53: $298-312$ 